\title{
La influencia de la comunicación comercial en el proceso decisional jerárquico: una evaluación empírica en el contexto educativo
}

\section{Gema Martínez Navarro}

Esic Business \& Marketing School

\section{Jaime Rivera-Camino}

Universidad Carlos III de Madrid Dpto. Economía de Empresa

Calle Madrid, 126

28903 Getafe (MADRID)

\section{Resumen}

A causa de la creciente competitividad a la que se enfrentan las universidades, éstas se ven obligadas usar la comunicación comercial en sus estrategias. Sin embargo, la literatura presenta vacíos conceptuales y empíricos que impiden su uso efectivo. Usando ecuaciones estructurales en una muestra de alumnos de una universidad pública, nuestra investigación trata de llenar esos vacíos. Después de una revisión bibliográfica se han desarrollado escalas con buenas propiedades psicométricas para evaluar un modelo que permite responder las siguientes preguntas: ¿Cuál es el proceso decisional que siguen los alumnos?, ¿Este proceso puede ser influido por las variables de comunicación comercial?, y ¿Cuál es la mejor forma de usar estas variables para conseguir los objetivos institucionales? Así, nuestra investigación contribuye a expandir la aplicabilidad de la comunicación comercial en un contexto no lucrativo y presenta líneas futuras de investigación en el tema. 
Palabras clave: comunicación comercial, proceso decisión compra, contexto universitario no lucrativo, competitividad empresarial.

Códigos JEL: M31, M37.

\section{INTRODUCCIÓN}

Amplia literatura coincide en señalar la importancia de la comunicación comercial para estimular la compra (Keller, 2001; Pickton, 2001) y para posicionar las ventajas competitivas de las empresas en mercados maduros (Belch y Belch, 2004, Duncan, 2005). Por este motivo, el estudio de su influencia en los compradores se ha considerado como el mayor desarrollo reciente en las comunicaciones (Kitchen et al., 2004). Pero, una revisión de la literatura también nos muestra que aún subsisten importantes vacíos que dificultan incrementar el estudio de esta influencia. Algunos autores indican que no se ha clarificado aún cual de los componentes de la comunicación comercial (publicidad, promociones, relaciones públicas) tiene más influencia en la compra de los consumidores (Balasubramanian, 1994; Swain, 2004). Otros autores señalan que casi no existen recomendaciones prácticas que puedan ayudar a incrementar la efectividad de la comunicación comercial (Cornelissen y Lock, 2000), porque aún no se han clarificado ciertos aspectos conceptuales de los componentes que la integran (Loda y Coleman, 2005). Otro vacío está relacionado a su escaso estudio en sectores no lucrativos, los cuales también requieren la aplicación de técnicas de comunicación comercial (Boffo, 2004).

Aunque existe una amplia tradición en analizar -de manera separada- la influencia de los componentes de la comunicación comercial (publicidad, promociones y relaciones públicas) en los compradores, sin embargo, aún son escasas las investigaciones que validen su impacto en sectores no lucrativos. Por tanto, en este sector aún no se conoce si las influencias de estos componentes pueden ser modificadas por factores individuales (e.g. proceso decisional), y/o por factores relacionados con su gestión.

El sector no lucrativo elegido para estudiar la aplicabilidad de la comunicación comercial es el sector universitario. Las razones que justifican nuestra elección son varias. En primer lugar, cada vez más se reconoce la influencia que tiene la educación superior en el desarrollo económico y social de los países (Ablett y Slengesol, 2000; Sinkovics y Schlegelmilch, 2000). En segundo lugar y a causa de su importancia, las universidades se ven obligadas a usar eficientemente la comunicación comercial para legitimizarse ante los diversos grupos de presión (Clark, 1998; Sen y Bhattacharya, 2001) y para captar alumnos y recursos para mantener su competitividad (Keaveney, 2001; Peterson y Hill, 1997). En tercer lugar, las universidades no pueden usar la comunicación comercial de la 
misma manera que las instituciones lucrativas. Por tanto, las universidades enfrentan la necesidad de contar con guías que les orienten sobre como usar la comunicación comercial para competir en este ambiente complejo, pero respetando sus características especificas de organizaciones no lucrativas. Para llenar estos vacíos y determinar si la comunicación comercial puede ser condicionada por factores individuales o de gestión, tres preguntas guían este estudio: (i) ¿Cuál es el tipo de proceso decisional que siguen los alumnos?, (ii) ¿Cuál componente de comunicación comercial influye más en este proceso?, (iii) ¿Es mayor la influencia de la Comunicación comercial cuándo se usan todos sus componentes, que cuándo se usa sólo el más efectivo de ellos?

Para responder a estas preguntas en la primera parte de nuestro artículo presentamos el marco teórico, así como las principales hipótesis derivadas del mismo. En esta parte revisamos los procesos de compra individual para justificar nuestra elección de estudiar los procesos decisionales jerárquicos. Igualmente justificamos la necesidad de clarificar la identidad de los principales componentes de la comunicación comercial antes de poder estudiar su efecto sobre el proceso decisional de compra. Después exponemos y comentamos los resultados y finalmente en las conclusiones, mencionamos nuestras contribuciones para futuras líneas de investigación.

\section{MARCO TEÓRICO Y ELABORACIÓN DE HIPÓTESIS}

\subsection{Los procesos decisionales de compra individual}

La literatura presenta diversos modelos para explicar las decisiones que siguen los consumidores en su proceso de elección y compra. Por ejemplo, el modelo tradicional sugiere la elección racional-económica, pero es criticado por autores que indican que las decisiones son condicionadas por la racionalidad limitada y los sesgos perceptuales de los decisores (Simon, 1992; Tversky y Kahneman, 1974). Los modelos motivacionales indican que la conducta de compra está influida por las necesidades que busca satisfacer, pero éstos son criticados por que son complejos y difíciles de validar empíricamente (Drenth et al., 1984). Los modelos modernos integran diferentes disciplinas y abordan el estudio del consumidor desde una perspectiva estadística (ver Lilien et al., 1992; Guadagni y Little, 1993), pero son criticados porque ignoran las variables subjetivas relacionadas a la compra (tales como la percepción, la motivación o la actitud). También se encuentran los modelos secuenciales que indican que el consumidor sigue un proceso o una serie de etapas que pueden ser influidas por las variables de comunicación comercial (ver Rossiter y Percy, 1997). Por tanto, aunque todos 
los modelos ofrecen guías sobre el proceso decisional de compra, no existe aún ninguno que sea ampliamente aceptado por los diversos autores. Los que presentan amplio poder conceptual explicativo, no han sido validados empíricamente. Y aquellos que han sido validados, no han integrado el efecto de las variables de comunicación comercial en un contexto educativo. Considerando que la mayoría coincide en asumir que el consumidor sigue un proceso secuencial, a continuación presentamos el modelo elegido, que se encuadra dentro de los modelos decisionales de respuesta jerárquica.

\subsection{Los Modelos decisionales de Respuesta Jerárquica}

Estos modelos se basan en sugerir una secuencia mental -o jerarquía de respuestas- a través de la cual el comprador pasa antes de generar una compra (Wells et al., 2003). Los antecedentes se encuentran en el clásico modelo AIDA de St. Elmo Lewis, que indica que el consumidor sigue cuatro etapas: atención, interés, deseo y acción. Dentro de este razonamiento, Lavidge y Steiner (1961), crearon un modelo para medir la efectividad de la publicidad. Este modelo sugiere que la conducta de compra está compuesta por tres categorías: el componente cognitivo o estado racional, el componente afectivo o estado emocional, y el componente conativo o estado motivacional. Otro ejemplo es Rogers (1962), quien afirma que los mensajes se difunden de manera jerárquica y presenta el proceso mental a través del cual un individuo adopta o compra regularmente un nuevo producto o servicio. Este modelo orienta las acciones de la comunicación y sugiere que el comprador sigue las siguientes etapas: conocimiento, interés, evaluación, prueba y adopción.

Basados en los conceptos de «cognitivo», «afectivo» y «conativo», Ray et al. (1973) propusieron un modelo con tres secuencias distintas, que puede explicar la respuesta de los consumidores ante la publicidad. Aquí se pueden distinguir tres modelos básicos. El primero es el modelo jerárquico clásico (Cognitivo/Afectivo/ Conativo) que tiene lugar cuando la audiencia requiere amplia información para un proceso racional de compra (con productos/servicios bien diferenciados). El segundo es el modelo de jerarquía atribución-disonancia, que funciona cuyo los productos/servicios tienen poca diferenciación y que sigue la secuencia: conativo, afectivo y cognitivo. El tercero, denominado jerarquía de bajo involucramiento desarrolla la secuencia: cognitivo, conativo y afectivo.

Estos componentes (Cognitivo/Afectivo/ Conativo) están representados en tres modelos de proceso de respuesta, tales como AIDA, jerarquía de efectos y procesa- 
miento de información (Belch y Belch, 2004; Christopher et al., 1995; Fill, 2005; Huizingh et al., 2003), los cuales asumen que el proceso se inicia con el conocimiento del producto, sigue con una evaluación favorable que motiva a la compra y culmina con la misma (Barry, 1987). Estos autores indican que cuando el consumidor desarrolla una compra racional (como la que cabe esperar de un alumno universitario), se sigue una ruta central de procesamiento de información (Myers, 2002) que se inicia con el proceso cognitivo (tal como postula el modelo jerárquico clásico). A continuación presentamos la Tabla 1 en la cual se muestra como este modelo permite resumir los aportes de diversos autores, sobre las diferentes etapas decisionales por las que pasa el comprador.

Tabla 1. Modelos de respuesta Jerárquica

\begin{tabular}{|c|c|c|c|c|c|}
\hline $\begin{array}{c}\text { MODELO } \\
\text { JERÁRQUICO } \\
\text { CLÁSICO }\end{array}$ & $\begin{array}{c}\text { AIDA } \\
\text { (Strong,1925) }\end{array}$ & $\begin{array}{c}\text { Lavidge y } \\
\text { Steiner (1961) }\end{array}$ & $\begin{array}{l}\text { Rogers } \\
(1961)\end{array}$ & $\begin{array}{c}\text { DAGMAR } \\
\text { Colley (1961) }\end{array}$ & $\begin{array}{c}\text { Howard y } \\
\text { Sheth (1969) }\end{array}$ \\
\hline Cognitivo & Atención & $\begin{array}{l}\text { Conciencia } \\
\text { Conocimiento }\end{array}$ & Conciencia & $\begin{array}{l}\text { Inconsciencia } \\
\text { Conciencia }\end{array}$ & 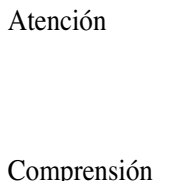 \\
\hline Afectivo & $\begin{array}{l}\text { Interés } \\
\text { Deseo }\end{array}$ & $\begin{array}{l}\text { Relación } \\
\text { Preferencia } \\
\text { Convicción }\end{array}$ & $\begin{array}{l}\text { Interés } \\
\text { Evaluación }\end{array}$ & Convicción & $\begin{array}{l}\text { Actitud } \\
\text { Intención }\end{array}$ \\
\hline Conativo & Acción & Compra & $\begin{array}{l}\text { Prueba } \\
\text { Adopción }\end{array}$ & Acción & Compra \\
\hline
\end{tabular}

Fuente: Elaboración propia.

Sin embargo, estos modelos no están exentos de críticas. Algunos autores indican que todos los compradores no atraviesan todas las etapas y que las compras por impulso contraen el proceso (Scholten, 1996), además que la secuencia de la respuesta jerárquica no está suficientemente validada (Huey, 1999; Weilbacher, 2001, 2002). Pero, otros autores defienden su valor para los académicos y directivos porque estos modelos son simples y lógicos, además que ayudan a predecir la compra, a pesar de sus imperfecciones (Barry, 2002; Barry y Howard, 1990). 
Considerando que los modelos jerárquicos han estado presente en la literatura de marketing durante más de 100 años (Weilbacher, 2001) y que son útiles para identificar la mejor estrategia de comunicación (Clow y Back, 2002), pero que aún no han sido suficientemente contrastados entre sí y menos aún en un ambiente educativo, desarrollamos las siguientes hipótesis alternativas:

H1a. Los alumnos universitarios siguen un proceso decisional jerárquico clásico. Por tanto, a mayor nivel del componente decisional Cognitivo, se encontrará un mayor nivel Afectivo y, a mayor nivel Afectivo se encontrará un mayor nivel Conativo.

H1b. Los alumnos universitarios siguen un proceso decisional de atribución-disonancia. Por tanto, a mayor nivel del componente decisional Conativo, se encontrará un mayor nivel Afectivo y, a mayor nivel Afectivo se encontrará un mayor nivel Cognitivo.

H1c. Los alumnos universitarios siguen un proceso decisional de bajo involucramiento. Por tanto, a mayor nivel del componente decisional Conativo, se encontrará un mayor nivel Cognitivo y, a mayor nivel Cognitivo se encontrará y un mayor nivel Afectivo.

\subsection{La comunicación comercial y el proceso decisional}

Aunque se han desarrollado varias definiciones sobre la comunicación comercial, aún no existen definiciones validadas empíricamente o que tengan amplia aceptación de los autores (Shimp y DeLoizier, 1986; Schultz et al., 1993). Según Blythe (2000), algunas definiciones prestan especial atención a la existencia de un elemento intencional en la comunicación comercial, pues indican que las empresas la usan para conseguir respuestas de sus mercados. Mientras que otras definiciones sugieren que la comunicación no es un proceso lineal puesto que un individuo puede comprar un producto por impulso y formarse una opinión de él tras la compra.

Al igual que ocurre con la comunicación comercial, la literatura nos presenta también diferentes maneras de definir las variables que la componen. Aunque algunos autores indican que existe cierta dificultad para separar y categorizar cada herramienta de la comunicación comercial (Smith, 1993), otros señalan que existen diferencias claras entre ellos (Arens, 1999; Batra et al, 1996). Así, encontramos publicaciones recientes que están dirigidas a estudiar las diferencias entre publicidad y relaciones públicas (ver Jo, 2004; Michaelson y Stacks, 2007; Van Reijmersdal et al., 2005; Weiner y Bartholomew, 2006), entre publicidad y promoción (Low y Mohr, 2000; 
Sethuraman y Tellis, 2002) y a sugerir la necesidad de diferenciar las relaciones públicas de las otras herramientas de marketing (Cheney y Christensen, 2001; Hutton, 2001).

Considerando que aún no está aclarado empíricamente cuales son los componentes de la comunicación comercial, para nuestra investigación hemos elegido los siguientes: la publicidad, la promoción y las relaciones públicas. La principal justificación para esta elección se haya en recientes publicaciones que indican que un plan de comunicación comercial debería incluir programas de publicidad, relaciones públicas y promoción de ventas (Benkahla, 2006; Wells et al., 2003).

\subsubsection{La influencia de la Publicidad en el proceso decisional}

Aunque la publicidad es definida como las comunicaciones comerciales que usan medios masivos y que se puede identificar al patrocinador (Hutton, 2002), la literatura indica que todavía existe cierta confusión sobre su identidad y sobre su efectividad. Por ejemplo, algunos autores vinculan la publicidad a las tareas propias de la disciplina del marketing ya que la restringen a comunicar hechos e imágenes sobre productos, servicios e ideas (Krugman et al., 1994). Otros autores también coinciden en indicar que la publicidad en ocasiones hace el trabajo del marketing, pero añaden que la publicidad puede afectarlo y es afectada por las variables económicas y sociales del mercado (Watson et al., 1990). En cuanto a su efectividad, algunos autores sugieren que la publicidad puede ser efectiva para impulsar a la compra, para buscar información, para modificar y/o reforzar actitudes (Bovee et al., 1995; Jones, 1992). Sin embargo, otros autores cuestionan la efectividad de la publicidad por que ésta toma mucho tiempo para incrementar las ventas y porque el retorno sobre la inversión es muy bajo (ver Abraham y Lodish, 1990).

La famosa expresión de que «la mitad del dinero gastado en publicidad es un desperdicio, pero no qué mitad» sugiere que examinar la efectividad de la publicidad debe ser un reto para los investigadores (Bovee et al.1995). Al respecto, los modelos de respuesta jerárquica de Lavidge y Steiner y McGuire asumen que la publicidad no impacta directamente la conducta, sino que previamente influye en la respuesta cognitiva y afectiva del consumidor. Así mismo, Vakratsas y Ambler (1999) después de una amplia revisión bibliográfica para entender como afecta la publicidad al consumidor, concluyeron que el aspecto cognitivo, afectivo y motivacional son tres medidas claves del efecto de la publicidad. Estos componentes del proceso decisional pueden ser usados como medida de respuesta ante los mensajes publicitarios y ayudar a estudiar 
aspectos no resueltos de los modelos jerárquicos (Barry, 2002). Aunque, al igual que Weilbacher $(2001,2002)$, indican que la influencia de la publicidad -según el modelo jerárquico- no ha sido validada empíricamente y que el tema debe ser estudiado aún más. Así considerado que no existen investigaciones orientadas a examinar la efectividad de la publicidad en el contexto educativo, y que no se ha clarificado su influencia en el proceso decisional de compra, proponemos la siguiente hipótesis:

H.2a. A mayor nivel de Publicidad, se encontrará un mayor nivel de la variable Cognitiva, Afectiva y Conativa.

H.2b. A mayor nivel de Publicidad se encontrará un mayor nivel del componente decisional Cognitivo. A mayor nivel del componente Cognitivo se encontrará un mayor nivel componente Afectivo y, a mayor nivel del componente Afectivo se encontrará un mayor nivel del componente Conativo.

\subsubsection{La influencia de la Promoción en el proceso decisional}

A pesar de la gran cantidad de dinero invertida en promoción, aún no se ha estudiado suficientemente su naturaleza y el proceso por el cual esta variable influye en el proceso decisional del consumidor (Belch y Belch, 2004). Respecto a su naturaleza, la promoción es un término reservado para las actividades que complementan a la publicidad y a la venta personal (Watson et al., 1990). Esta definición es ampliada por algunos autores que mencionan que la promoción incluye todas las formas de comunicación -excepto relaciones públicas, venta personal o publicidad- que llaman la atención sobre un producto y/o refuerzan el mensaje de la publicidad (Krugman et al., 1994). En cuanto a su operacionalización, la promoción consta de diversas actividades (tales como el cambio de cupones, muestras gratuitas, los dos por uno, etc..) diseñadas para dar un impulso temporal a las ventas (Blythe, 2000). Aunque también se indica que existen varios tipos de promociones, las que se basan en incentivos y están orientadas al precio y las que se basan en la comunicación y ofrecen una razón a corto plazo para la compra (Kotler et al., 1999; Tellis, 1998).

En cuanto a su influencia, la literatura coincide en que esta variable busca impulsar la compra de un producto o servicio (Smith, 1993) porque afecta la percepción de precio, valor e intención de compra de los compradores (Munger y Grewal, 2001). Aunque también se encuentra que esta influencia puede estar condicionada por la categoría de producto y por variables internas del comprador. Así, el 90\% de los bienes de consumo masivo pueden ser vendidos con ayuda de promociones (Abraham y Lodish, 
1987), pero también, ciertos bienes y servicios pueden ver afectada su calidad si se usan promociones en su venta (Ong, 1997). En cuanto a las variables internas, las promociones influyen en los compradores que usan criterios de utilidad racional económica (Groth y Dye, 1999; Quock y Uncles, 2005) y que tienen poca experiencia de compra (Low y Mohr, 2000). Aunque algunos autores agregan que las promociones influyen en los compradores que mezclan criterios económicos y hedonistas (Chandon et al., 2000).

La literatura también indica que la influencia positiva de la promoción para incrementar la tendencia a la compra se ha estudiado en varios sectores. Por ejemplo, en la promoción de destinos turísticos (Dore y Crouch, 2003), de conductas saludables (Jaeger, 2001; McKay, 2003), en el incremento de la imagen de marca (Graeff, 1996) y de la intención de compra (Chen, 1998; Smith y Vogt, 1995), y en la aceptación de organizaciones no lucrativas (Bennett y Kottasz, 2006). Sin embargo, también se encuentra que aún no se ha estudiado suficientemente el impacto de la promoción en las etapas de la jerarquía de respuestas. Una excepción es Gardener y Trivedi (1998), quienes sugirieron que la promoción funciona cuando ésta pasa por todos las etapas del proceso jerárquico, aunque estos autores no validaron empíricamente sus afirmaciones. Por tanto, desarrollamos las siguientes hipótesis:

H.3. A mayor nivel de Promoción se encontrará un mayor nivel del componente decisional Cognitivo. A mayor nivel del componente Cognitivo se encontrará un mayor nivel componente Afectivo y, a mayor nivel del componente Afectivo se encontrará un mayor nivel del componente Conativo.

\subsubsection{La influencia de las Relaciones Públicas en el proceso decisional}

Tradicionalmente, las relaciones públicas se han definido como una forma de obtener publicidad gratuita a través de los contactos con los medios, sin embargo, recientemente los directivos defienden que esta variable es más amplia que sólo la publicidad (Gregory, 2001). Así, todavía existe cierta discusión sobre su definición y sobre su efectividad.

Para algunos autores, las relaciones públicas pueden sustituir a la publicidad como elemento importante en la mezcla de comunicación comercial (Harris 1993; Ries y Ries, 2002). Para otros autores, las relaciones públicas no deberían ser consideradas como una función de marketing y por tanto no deberían ser integradas en la comuni- 
cación comercial (Lauzen, 1991). A pesar de esta discusión, una de las definiciones más aceptadas indica que las relaciones públicas es la función directiva que establece y mantiene relaciones benéficas entre una organización y los públicos de los cuales depende para su funcionamiento institucional (Cutlip et al., 2000). Por este motivo, es una variable especialmente recomendada para las organizaciones no lucrativas, porque éstas pueden hacer dudar de su legitimidad moral si invierten dinero en otras actividades de comunicación (Donnelly-Cox y O’Regan, 1999).

En cuanto a su efectividad, los autores coinciden en señalar que las relaciones públicas influyen en la compra porque apoyan las acciones de las otras variables de la comunicación comercial. Según Shimp (1993) las relaciones públicas también alientan a la compra porque promocionan la buena voluntad de la empresa y la buena imagen de los productos. Según Kotler (1996), si las relaciones públicas usan noticias y comunicaciones en un contexto editorial, pueden ayudar a la credibilidad de los beneficios del producto, apoyar a la fuerza de ventas y entusiasmar al distribuidor. Aunque hay ciertas diferencias sobre cuales son los criterios que se deberían usar para evaluar la efectividad de las relaciones públicas (ver Holloway, 1992; Phillips, 2001), se recomienda evaluar su impacto en la comprensión del mensaje (elemento cognitivo), así como en los cambios actitudinales y conductuales derivados del mismo (Lindenmannn, 1993).

La revisión de la literatura muestra que las relaciones públicas han sido estudiadas en diferentes sectores y aplicaciones. Por ejemplo, en el posicionamiento de marcas (Pullig et al., 2006), en la aceptación de políticas públicas (Thorsten, 2007), en la mejora de imagen corporativa (Renkema y Hoeken, 1998). En el sector educativo, aunque se ha investigado su uso en el reclutamiento de estudiantes minusválidos (Ralph y Boxall, 2005), las publicaciones relacionadas a nuestro tema de estudio aún son escasas. Así, elaboramos la siguiente hipótesis:

H.4. A mayor nivel de Relaciones Públicas se encontrará un mayor nivel del componente decisional Cognitivo. A mayor nivel del componente Cognitivo se encontrará un mayor nivel componente Afectivo y, a mayor nivel del componente Afectivo se encontrará un mayor nivel del componente Conativo. 


\subsection{La influencia diferencial de los componentes en el proceso decisional}

Aunque la literatura revisada anteriormente sugiere que los componentes de la comunicación comercial pueden influir en el proceso decisional, sin embargo, todavía no se conoce cual de ellos es el más eficiente para el contexto de organizaciones educativas. El panorama conceptual encontrado es aún desintegrado, escaso y con resultados poco concluyentes. Por ejemplo, diversos autores sugieren que las relaciones públicas son más creíbles, persuasivas y efectivas que la publicidad (ver Ray, 1994; Ries y Ries, 2002). Sin embargo, se recomienda cierta precaución en la aceptación de estas afirmaciones dado que existe aún poca investigación empírica al respecto (Hunt y Grunig, 1993). Esta desconfianza se mantiene porque una revisión de 12 estudios experimentales indica que dos estudios concluyeron que relaciones públicas son más creíbles que la publicidad, siete no fueron concluyentes y que tres tuvieron problemas metodológicos que alteraron los resultados (Hallahan, 1999). Igualmente, Dore y Crouch (2003) después de realizar una investigación exploratoria para analizar el impacto diferencial entre relaciones públicas y promociones concluyeron que se requiere investigar todavía más el tema. Por otra parte, Smith y Vogt (1995) compararon la efectividad de la publicidad con el boca-boca en el sector turístico, sólo para concluir que es necesario un mayor estudio para investigar como integran los consumidores la publicidad con otras variables de comunicación. Dado que todavía no se conoce suficientemente cual de los componentes tiene más influencia en el proceso decisional (Balasubramanian, 1994; Swain, 2004), y menos aún en un contexto educativo, desarrollamos las siguientes hipótesis alternativas:

H.5a. La Publicidad tiene mayor influencia que la Promoción y las Relaciones Públicas en el proceso decisional jerárquico.

H.5b. La Promoción tiene mayor influencia que la Publicidad y las Relaciones Públicas en el proceso decisional jerárquico.

H.5c. Las Relaciones Públicas tienen mayor influencia que la Publicidad y la Promoción en el proceso decisional jerárquico. 


\subsection{El efecto de la comunicación comercial integrada (CCI) en el proceso decisional}

Reciente literatura sugiere que la base de una estrategia de comunicación comercial exitosa es el uso coordinado de sus componentes (Hutton, 2002; Clow, 2002), y que se debe evitar aplicarlos de forma separada (Dwyer y Tanner, 2002). Aunque en esto coinciden directivos y académicos, también se reconoce la dificultad de evaluar la efectividad de su uso conjunto (Schultz y Kitchen, 2000). Por tanto, se requiere investigar si la gestión combinada de los componentes de comunicación comercial (comunicación comercial integrada-CCI) es más efectiva, que el uso separado de los mismos (Loda y Coleman, 2005). Esta efectividad generada por el uso combinado de los componentes puede ser explicada por la literatura de procesamiento de información, la cual sugiere que los decisores integran información cuando desarrollan ciertos procesos mentales. Por ejemplo, Anderson (1981) indica que la evaluación de ideas o cosas requiere que los decisores integren información proveniente de diferentes fuentes. Igualmente, Smith y Swinyard (1982) también proponen que la formación de actitudes demanda la integración de diversas fuentes de comunicación.

Esta idea de «integración» también se ha encontrado presente en los directivos de empresas, desde la década de los 80s (Duncan y Caywood, 1996). Por tanto, Schultz et al., (1993) señalan que la meta de la comunicación comercial es desarrollar programas que se complementen entre si, para que faciliten la integración de la información destinada a promover la conducta de compra de los clientes. Esta afirmación puede ser apoyada por autores que señalan que el efecto de las relaciones públicas no se puede estudiar de manera aislada, y que su impacto en los consumidores pasa por integrar programas de publicidad y promociones (Moriarty, 1994). Así, se demanda estudiar la sinergia, en donde un componente de la comunicación comercial refuerza el impacto del otro y como resultado se obtiene un efecto mayor que el impacto aislado de cada componte (Naik y Raman, 2003; Shih-Lun y Nelson, 2006; Weilbacher, 2001). Aunque algunos autores han investigado la sinergia entre medios y radio, televisión e internet (Edell y Keller, 1999; Chang y Thorson, 2004), sin embargo aún son escasas las investigaciones sobre el efecto de combinar diferentes componentes de comunicación, tales como publicidad y relaciones públicas (Jin, 2003). Considerando que todavía existen pocas investigaciones al respecto (Naik y Raman, 2003; Weilbacher, 2001) -y que este vacío es mayor en el sector educativo-, desarrollamos la siguiente hipótesis: 
H.6. La influencia de la comunicación comercial en el proceso decisional es mayor cuando se usan todos sus componentes (CCI), que cuando se usa aisladamente el más efectivo de ellos.

\section{ANÁLISIS EMPÍRICO Y RESULTADOS}

\subsection{Descripción de la muestra}

Nuestra población objetivo estuvo compuesta de 4.950 alumnos provenientes de una universidad pública de Madrid. De esta población objetivo se obtuvieron 580 cuestionarios, de los cuales se determinaron 563 como válidos $(11,37 \%)$. La tabla 2 se presenta los detalles de la muestra.

Tabla 2. Descripción de la muestra por titulación

\begin{tabular}{|l|c|c|c|c|c|c|c|c|c|}
\hline VARIABLES & \multicolumn{9}{|c|}{ LICENCIATURA } \\
\hline & LADE & $\begin{array}{c}\text { LADE }+ \\
\text { DCHO }\end{array}$ & $\begin{array}{c}\text { ECO + } \\
\text { DCHO }\end{array}$ & $\begin{array}{c}\text { CC } \\
\text { TRABAJO }\end{array}$ & PERIOD. & SOCIOL. & HUMAN. & TOT. & $\%$ \\
\hline $\begin{array}{l}\text { EDAD } \\
18-20\end{array}$ & 0 & 0 & 0 & 9 & 6 & 19 & 10 & 44 & 7,8 \\
\hline $21-23$ & 98 & 51 & 53 & 54 & 60 & 58 & 54 & 428 & 76 \\
\hline+24 & 15 & 20 & 19 & 18 & 6 & 4 & 9 & 91 & 16,1 \\
\hline TOTAL MUESTRA & 113 & 71 & 72 & 81 & 72 & 81 & 73 & 563 & 100 \\
\hline $\begin{array}{l}\text { TOTAL POBLACIÓN } \\
\%\end{array}$ & 960 & 840 & 860 & 620 & 720 & 480 & 470 & 4950 & \\
\hline \hline $\begin{array}{c}\text { SEXO } \\
\text { H }\end{array}$ & 11,77 & 8,45 & 8,37 & 13,06 & 10,00 & 16,88 & 15,53 & & \\
\hline M & 30 & 51 & 48 & 53 & 47 & 44 & 46 & 342 & 60,7 \\
\hline TOTAL & 113 & 71 & 72 & 81 & 72 & 81 & 73 & 563 & 100 \\
\hline
\end{tabular}

Aunque en nuestra muestra incluimos encuestados con diferencias en cuanto a sexo y edad, sin embargo, en la investigación integramos sus respuestas en una sola muestra. La razón de no usar submuestras es por que en un análisis previo encontramos que no existían diferencias significativas $(\mathrm{p}>0.93 ; \mathrm{p}>0.87$, respectivamente) 
entre estas variables respecto a la variable dependiente en estudio (Conativa). Las tablas 3 y 4 describen los resultados del análisis mencionado.

Tabla 3. Dependiente conativo, independiente sexo

\begin{tabular}{|l|c|c|c|c|c|}
\hline \multicolumn{1}{|c|}{ SEXO } & $\begin{array}{c}\text { Suma } \\
\text { cuadrados }\end{array}$ & gl & $\begin{array}{c}\text { Medias } \\
\text { cuadrát. }\end{array}$ & $\mathrm{F}$ & Sig. \\
\hline Inter- & .00 & 1 & .00 & .00 & .93 \\
\hline Intra- & 608.40 & 561 & 1.08 & & \\
\hline Total & 608.41 & 562 & & & \\
\hline
\end{tabular}

Tabla 4. Dependiente conativo, independiente edad

\begin{tabular}{|c|c|c|c|c|c|c|}
\hline \multicolumn{2}{|c|}{ EDAD } & \multirow{2}{*}{$\begin{array}{c}\text { Diferencia de } \\
\text { medias }\end{array}$} & \multirow[t]{2}{*}{ Error } & \multirow[t]{2}{*}{ Sig. } & \multicolumn{2}{|c|}{ Intervalo de conf. $95 \%$} \\
\hline (I) & $(\mathrm{J})$ & & & & Límite & Límite sup. \\
\hline $18-20$ & $\begin{array}{l}21-23 \\
\text { más de }\end{array}$ & $\begin{array}{l}-, 0096 \\
-, 0712\end{array}$ & $\begin{array}{l}, 1649 \\
, 1913\end{array}$ & $\begin{array}{l}, 998 \\
, 933\end{array}$ & $\begin{array}{l}-, 4145 \\
-, 5408\end{array}$ & $\begin{array}{l}, 3953 \\
, 3984\end{array}$ \\
\hline $21-23$ & $\begin{array}{l}\text { 18-20 } \\
\text { más de }\end{array}$ & $\begin{array}{c}, 0096 \\
-, 0616\end{array}$ & $\begin{array}{l}, 1649 \\
, 1202\end{array}$ & $\begin{array}{l}, 998 \\
, 877\end{array}$ & $\begin{array}{l}-, 3953 \\
-, 3569\end{array}$ & $\begin{array}{l}, 4145 \\
, 2336\end{array}$ \\
\hline más de & $\begin{array}{l}18-20 \\
21-23\end{array}$ & $\begin{array}{l}, 0712 \\
, 0616\end{array}$ & $\begin{array}{l}, 1913 \\
, 1202\end{array}$ & $\begin{array}{l}, 933 \\
, 877\end{array}$ & $\begin{array}{l}-, 3984 \\
-, 2336\end{array}$ & $\begin{array}{l}, 5408 \\
, 3569\end{array}$ \\
\hline
\end{tabular}

Para poder inferir nuestros resultados a la población y presentar aportes a los estudios que se realicen con posterioridad, es importante analizar si el tamaño de nuestra muestra es representativo. El procedimiento usado para analizar el tamaño de la muestra se basa en Lambin (1994), quien indica si se conoce la máxima desviación estándar de las variables de nuestro modelo, se puede calcular el tamaño mínimo de la muestra, con cierto margen de error aceptable. El supuesto de base es que el tamaño de la muestra obtenido permitirá justificar las variables con la desviación estándar más pequeñas. La fórmula establece lo siguiente:

\section{Tamaño de muestra $=($ Nivel de significación $x$ Desviación estándar error aceptable $)^{2}$}

En nuestro caso, la variable con la mayor desviación estándar es Interés en recibir información de esta universidad (Conativo-3), con las siguientes características: 
Promedio (3,26); Desviación Estándar $(1,24)$; Error típico $(0,6)$. Aplicando la fórmula propuesta por Lambin (1994), para todas las variables consideramos el mismo nivel de significancia $(0,01)$ y un error de 0,15 (en una escala de 1 a 5). Por tanto, aplicando la fórmula anterior, una muestra de 322 alumnos sería suficiente.

\subsection{Cuestionarios}

A continuación presentamos las escalas utilizadas para evaluar cada una de las variables de nuestro modelo. Dado la originalidad de nuestra investigación y la escasez de investigaciones similares, todas las escalas siguieron un procedimiento similar: revisión de la literatura, elaboración de ítems, discusión con profesores de marketing y con directivos con experiencia en comunicación comercial, estudio piloto, y depuración de ítems para mejorar la fiabilidad y validez de las escalas. El estudio piloto se desarrolló con todos los ítems iniciales en una muestra de conveniencia de 111 alumnos de LADE, LADE/Derecho y LADE-Economía (35, 40, 36 alumnos, respectivamente). Estos ítems iniciales fueron desarrollados a partir de la revisión de la literatura usada en el marco teórico de las hipótesis.

En esta etapa se eliminaron los ítems con menor correlación interna $(<0,50)$ al interior de cada variable. Posteriormente se realizó un análisis factorial exploratorio entre todas las variables que intervienen en nuestro modelo (análisis de componentes principales, método de rotación Varimax). El objetivo de este análisis fue discriminar entre los ítems que aparecían agrupados en el factor o variable prevista, y aquellos que participaban en varios factores. El propósito fue eliminar posteriormente a estos últimos, para evitar problemas en la estimación de los parámetros y en el análisis con ecuaciones estructurales. Estos procesos explican porque se eliminaron varios ítems de aquellos que inicialmente fueron desarrollados con ayuda de la teoría. La validez de los diferentes constructos usados en nuestro modelo (que es presentada más adelante) justifica el éxito del proceso de depuración de las escalas.

\section{- La variable Cognitiva}

Esta variable fue medida inicialmente por medio de diecisiete indicadores, que evaluaban el conocimiento de determinados atributos de la Universidad tales como la imagen frente a la competencia, las adecuadas instalaciones, la calidad de los profesores, entre otros. Después del proceso de depuración, quedaron cuatro ítems que fueron evaluados con una escala de intervalo, donde: (1) Nada de acuerdo, (3) Algo de acuerdo y (5) Muy de acuerdo. La fiabilidad de esta escala fue de 0.91 (Alfa Cronbach). 


\section{- La variable Afectiva}

Para evaluar esta variable se generaron inicialmente dieciséis ítems que trataban de medir la satisfacción o insatisfacción de los alumnos con determinadas características y acciones de la Universidad. Por ejemplo, la imagen de la Universidad ante la sociedad, las acciones de comunicación comercial que realiza la Universidad o los logros personales que ha conseguido en esta Universidad. La escala final, compuesta de cinco ítems usó una escala de 1 a 5. En donde (1) Nada de acuerdo, (3) Algo de acuerdo y (5) Muy de acuerdo. La fiabilidad de la escala fue de 0.92.

\section{- La variable Conativa}

Inicialmente se generaron nueve ítems para medir esta variable. La escala final estuvo compuesta de cuatro ítems que evaluaban la probabilidad de volver a adquirir algunos servicios de la Universidad así como de recomendarla a otros. Por ejemplo: Aconsejaría a sus amigos estudiar en esta Universidad, Se matricularía en otros cursos de esta Universidad, Desearía ser miembro colaborador en las actividades de esta Universidad, Volvería a tomar la decisión de cursar aquí sus estudios, etc. Para evaluar esta variable también se usó una escala de intervalo que medía de menos a más el grado en que se cumpliría dicha proposición, donde 1 «Nada» y 5 «Mucho». La fiabilidad de la escala fue de 0.88 .

\section{- La variable Relaciones Públicas}

Los ítems fueron derivados de las diversas operacionalizaciones que presentan los autores (Arens, 1999; Blythe, 2000; Cutlip et al., 1994; Grunig, 1993 ; Krugman et al., 1994; O’Guinn et al., 1990; Shimp, 1993; Smith, 1993). Para analizar las acciones de relaciones públicas que desarrolla la universidad se desarrolló un cuestionario inicial compuesto por quince ítems, los cuales recogían las principales acciones que son mencionadas por la teoría. Como por ejemplo, Realiza acciones de prensa, Charlas para favorecer la calidad de vida personal del estudiante: control del estrés, técnicas de estudio, Relaciones con otras instituciones y empresas patrocinadoras, Guías de la Universidad con información de interés general (estatutos, órganos de gobiernos...) ó Establece relaciones con profesionales y personalidades de reconocido prestigio. La escala final de cuatro ítems se graduó con las mismas características que las anteriores variables, es decir con un intervalo de 1 a 5 . En donde (1) Nada de acuerdo, (3) Algo de acuerdo y (5) Muy de acuerdo. La fiabilidad de la escala fue de 0.89.

\section{- La variable Publicidad}

Los ítems que permiten operacionalizar la variable publicidad son diversos y son 
varios los autores que coinciden en señalarlos (Arens, 1999; Blythe, 2000; O'Guinn et al., 1990), aunque en nuestro caso, debimos adaptarlos al contexto educativo. Las acciones de publicidad que desarrolla la Universidad se midieron inicialmente con un cuestionario de catorce ítems. El cuestionario final de cinco ítems usó una escala graduada de 1 a 5, para medir el nivel de acuerdo o desacuerdo del alumno con las proposiciones presentadas en la escala, las cuales presentaba las diversas acciones, medios usados y resultados obtenidos por la universidad. Por ejemplo: Utiliza anuncios en prensa para informar de sus características y productos/servicios institucionales, Da la posibilidad de adquirir productos relacionados con la Universidad (relojes, corbatas, pins, llaveros, camisetas, bolígrafos,...), Tiene una marca que identifica a la Universidad con características muy definidas ó Tiene un slogan. La fiabilidad de la escala fue de 0.79.

\section{- La variable Promoción}

Se tomaron en cuenta los principales ítems a través de los que se operacionaliza la variable promoción (Arens, 1999; Blythe, 2000; Krugman et al., 1994; O’Guinn et al., 1990; Rivera et al., 2002 ; Shimp, 1993; Smith, 1993; Watson et al., 1990), para adaptarlos al contexto educativo. Así, para evaluar las acciones promocionales y los medios utilizados por la Universidad se utilizó un cuestionario compuesto inicialmente por trece ítems. Por ejemplo, La Universidad acude a ferias donde promociona la educación universitaria, Realiza eventos y espectáculos con precios especiales para los universitarios, Facilita la obtención de tarjetas que permiten descuentos y el acceso a diferentes servicios de la Universidad (tarjeta monedero) ó Promociona la investigación académica. El cuestionario final de cuatro ítems obtuvo una fiabilidad de 0.84 y usó una escala de 1 a 5 para determinar el grado de acuerdo o desacuerdo con las proposiciones presentadas, en donde: (1) Nada de acuerdo, (3) Algo de acuerdo y (5) Muy de acuerdo.

\section{ANÁlisis y RESUltados}

En la primera parte se expone el tratamiento estadístico seguido para evaluar la validez de los constructos y de las hipótesis. Aquí también se presenta el procedimiento denominado evaluación de modelos rivales que es el apropiado para comparar hipótesis alternativas ( $\mathrm{H} 1$; H5; H6). Asimismo, se muestran los índices usados para evaluar el grado en el cual los datos se ajustan a los modelos estructurales (H2; H3; H4) y que también se han utilizado para analizar las mejoras o desarrollo de los modelos evaluados 


\subsection{Análisis estadístico}

De acuerdo a la recomendación de Anderson y Gerbin (1988), inicialmente desarrollamos un análisis en dos etapas. En la primera etapa, y para confirmar los modelos de medida evaluamos si las variables observadas están relacionadas a las variables latentes. En la segunda etapa se validó el grado en el cual los datos se ajustan a los modelos estructurales. Para evaluar tanto el modelo de medida como el estructural usamos los siguientes índices: el SRMR (Standardised Root Mean Square Residual: Steiger, 1990); GFI y AGFI (Goodness-of-Fit Index, Adjusted Goodness of Fit Index: Jöreskog y Sörbom, 1993); CFI (Comparative Fit Index: Bentler, 1990) y el IFI (Incremental Fit Index: Bollen, 1989). No usamos el test Chi cuadrado $\left(\mathrm{X}^{2}\right)$ por su sensibilidad a muestras grandes (Cheung y Rensvold, 2002). Siguiendo la práctica comúnmente aceptada, un GFI mayor de 0.90 y un SRMR 0.08 o menor, fueron considerados como indicadores de bondad de ajuste del modelo especificado (Hatcher, 1994). Para comparar los modelos se usó un procedimiento denominado evaluación de modelos rivales (Bollen y Long, 1992) en donde se pretende mostrar que el modelo propuesto se ajusta mejor a los datos que otros modelos alternativos o rivales (Kelloway, 1998). Para esta comparación se desestimó el uso de la razón Q de Joreskög (1979) porque ésta se basa en $X^{2}$ y se usó el procedimiento recomendado por Cheung y Rensvold (2002). Estos autores indican que un incremento de 0.01 o más, en el índice CFI señala una contribución significativa de la nueva relación incluida en el modelo. Para la estimación de los modelos usamos la matriz de correlación de productomomento (Pearson). Se eligió este tipo de matriz porque es conveniente cuando se desea comparar los coeficientes al interior de un modelo y porque es invariante ante cambios de escala, es decir, no da mayor peso a aquellas variables que poseen mayor variabilidad, de manera que no se distorsionan los resultados. Asimismo, se usó el método interactivo de mínimos cuadrados ponderados, puesto que éste no necesita el supuesto de normalidad.

\subsection{Test de validez de constructos}

Antes de analizar la bondad de ajuste de los modelos se investigaron varios tipos de validez de constructo. El Análisis Factorial Confirmatorio se usó para evaluar si los ítems sirven como elementos descriptivos y de medida de las variables latentes (Bollen, 1989; Jöreskog, 1993). La tabla 5 muestra que todos los ítems considerados 

JERÁRQUICO: UNA EVALUACIÓN EMPÍRICA EN EL CONTEXTO EDUCATIVO

Tabla 5. Validez de Contenido y Convergente

\begin{tabular}{|c|c|c|c|c|c|}
\hline Variable Latente & Coefic. & $\begin{array}{c}\mathrm{t}- \\
\text { Student }\end{array}$ & $\begin{array}{l}\text { Variz } \\
\text { Error }\end{array}$ & $\mathrm{R}^{2}$ & GFI \\
\hline COGNITIVO & & & & & 0.98 \\
\hline 1. Facilita la inserción laboral & 0.62 & 14.24 & 0.61 & 0.38 & \\
\hline 2. Recursos suficientes para facilitar la enseñanza & 0.65 & 14.96 & 0.58 & 0.42 & \\
\hline 3. Buena imagen frente a la competencia & 0.64 & 14.64 & 0.59 & 0.41 & \\
\hline 4. Constante actualización de profesores & 0.69 & 16.18 & 0.52 & 0.48 & \\
\hline 5. Buena imagen frente mundo empresarial & 0.78 & 19.07 & 0.39 & 0.61 & \\
\hline 6. Ser sinónimo de calidad & 0.74 & 17.79 & 0.45 & 0.55 & \\
\hline AFECTIVO & & & & & 0.98 \\
\hline 1. Imagen de la universidad ante la sociedad & 0.58 & 11.12 & 0.66 & 0.34 & \\
\hline 2. Medios utilizados para hacer llegar la información & 0.67 & 12.12 & 0.55 & 0.45 & \\
\hline 3. Tipo de información que hace llegar a los alumnos & 0.72 & 13.34 & 0.48 & 0.52 & \\
\hline 4. Forma de manejar la imagen de los alumnos & 0.71 & 12.43 & 0.49 & 0.50 & \\
\hline 5. Impacto causado en el alumno & 0.57 & 10.51 & 0.69 & 0.32 & \\
\hline 6. Acciones frente a otras universidades & 0.59 & 11.17 & 0.66 & 0.35 & \\
\hline CONATIVO & & & & & 0.99 \\
\hline 1. Aconsejaría estudiar en esta universidad & 0.77 & 13.22 & 0.31 & 0.59 & \\
\hline 2. Participar en la asociación antiguos alumnos & 0.68 & 9.72 & 0.49 & 0.46 & \\
\hline 3. Interés en recibir información de esta universidad & 0.85 & 13.63 & 0.29 & 0.72 & \\
\hline 4. Colaborar en las actividades de esta universidad & 0.69 & 11.51 & 0.47 & 0.48 & \\
\hline 5. Volvería a cursar aquí los estudios & 0.73 & 12.76 & 0.44 & 0.53 & \\
\hline PROMOCIÓN & & & & & 1 \\
\hline 1. Clubs y asociaciones de alumnos & 0.65 & 12.62 & 0.55 & 0.42 & \\
\hline 2. Oficinas de información & 0.61 & 10.71 & 0.62 & 0.37 & \\
\hline 3. Ferias promoción de educación universitaria & 0.75 & 14.93 & 0.44 & 0.56 & \\
\hline 4. Tarjetas que permiten obtener descuentos & 0.66 & 14.07 & 0.54 & 0.44 & \\
\hline RRPP & & & & & 0.98 \\
\hline 1. Charlas para favorecer inserción laboral & 0.67 & 11.85 & 0.56 & 0.45 & \\
\hline 2. Guías de la universidad con información de interés & 0.68 & 12.11 & 0.54 & 0.46 & \\
\hline 3. Becas que repercutan en el bienestar social & 0.65 & 10.14 & 0.57 & 0.42 & \\
\hline 4. Comunicaciones de bienestar social & 0.72 & 14.16 & 0.43 & 0.52 & \\
\hline 5. Jornadas de puertas abiertas & 0.71 & 13.41 & 0.45 & 0.50 & \\
\hline 6. Acciones formativas en zonas deprimidas & 0.61 & 9.76 & 0.63 & 0.37 & \\
\hline PUBLICIDAD & & & & & 0.99 \\
\hline 1. Se venden productos de la universidad & 0.63 & 11.14 & 0.55 & 0.40 & \\
\hline 2. La universidad tiene un slogan claro & 0.59 & 9.01 & 0.64 & 0.35 & \\
\hline 3. Posee un archivo fotográfico de instalaciones & 0.69 & 13.51 & 0.49 & 0.48 & \\
\hline 4. Permite acceder a los CV de sus profesores & 0.73 & 14.60 & 0.44 & 0.53 & \\
\hline 5. Posee catálogos donde publicita su oferta educativa & 0.65 & 12.56 & 0.54 & 0.42 & \\
\hline
\end{tabular}


en los modelos estructurales fueron significativos y mostraron un aceptable nivel de $\mathrm{R}^{2}$ y de GFI. La validez convergente fue analizada a base de la significancia de los coeficientes de regresión, según la recomendación de Anderson y Gerbing (1988). De acuerdo a los coeficientes mostrados en la tabla 2, tanto la validez de contenido como la convergente se encontraron en todos los seis constructos.

\subsection{Validación de hipótesis}

La Hipótesis 1 trata de evaluar cual de los procesos jerárquicos explica mejor el proceso decisional de los componentes de nuestra muestra. De acuerdo a los índices y parámetros mostrados en la tabla 6 se confirma la hipótesis H.1 ${ }^{\mathrm{a}}$ que establece que los alumnos siguen un modelo jerárquico clásico o racional en sus decisiones. El modelo clásico presenta -ver tabla 7- un ajuste absoluto superior a los otros modelos (GFI $=0.93$; AGFI $=0.90)$, la apreciación de los residuos es menor $(\mathrm{SRMR}=0.04)$ y los índices incrementales también son mayores (CFI e IFI $=0.95)$. En la tabla 7 se muestran los resultados finales del modelo jerárquico clásico.

Tabla 6. Evaluación de modelos rivales

\begin{tabular}{|l|c|c|c|}
\hline \multicolumn{1}{|c|}{ Indicadores } & Modelo clásico & Modelo disonancia & Modelo bajo involuc. \\
\hline - GFI & 0.93 & 0.84 & 0.83 \\
\hline - AGFI & 0.90 & 0.79 & 0.78 \\
\hline - SRMR & 0.042 & 0.097 & 0.10 \\
\hline - CFI & 0.95 & 0.87 & 0.85 \\
\hline - IFI & 0.95 & 0.87 & 0.85 \\
\hline
\end{tabular}

Tabla 7. Resultados del modelo final del modelo clásico

\begin{tabular}{|c|c|c|c|c|}
\hline $\begin{array}{c}\text { Variables } \\
\text { dependientes }\end{array}$ & Coeficientes & $\mathrm{T}$ & $\begin{array}{c}\text { Varianza } \\
\text { del error }\end{array}$ & $\mathrm{R}^{2}$ \\
\hline $\begin{array}{c}\text { AFECTIVA } \\
\text { - Cognitiva }\end{array}$ & 0.88 & 12.82 & 0.23 & 0.77 \\
\hline $\begin{array}{c}\text { CONATIVA } \\
\text { Afectiva }\end{array}$ & 0.84 & 11.01 & 0.30 & 0.70 \\
\hline
\end{tabular}


La hipótesis 2 trata de analizar el impacto de la publicidad en la secuencia jerárquica decisional de los alumnos. Se evaluó el modelo inicial y los indicadores señalaron que nuestro modelo presentaba un ajuste aceptable a los datos disponibles $(\mathrm{SRMR}=0.065 ; \mathrm{GFI}=0.91 ; \mathrm{AGFI}=0.88 ; \mathrm{CFI}=0.93 ; \mathrm{IFI}=0.93)$.

Se revisó la literatura para analizar si existían fundamentos teóricos para agregar nuevas relaciones entre las variables y conseguir un mejor ajuste. La literatura muestra que la manipulación en el componente afectivo puede influir en la efectividad de los mensajes publicitarios (De Pelsmacker et al., 2002; Fabrigar y Petty, 1999), pues altera la ruta -central o periférica- que se usa para su procesamiento (Aylesworth y MacKenzie, 1998) e impacta en el recuerdo e interpretación de los mismos (Sharma, 2000; Yi, 1990).

Después de incorporar la relación Publicidad-Afectiva se encontró que el nuevo modelo ofrecía un mejor ajuste $(\mathrm{SRMR}=0.060 ; \mathrm{GFI}=0.92 ; \mathrm{AGFI}=0.90 ; \mathrm{CFI}=0.94$; $\mathrm{IFI}=0.94)$. Los índices y parámetros mostrados en la tabla 8 , confirman que a mayor publicidad, se encontrará un mayor nivel de los componentes cognitivo, afectivo y conativo, pero con la influencia mediadora de la variable emocional o afectiva.

Tabla 8. Resultados del modelo final de la influencia de la Publicidad

\begin{tabular}{|c|c|c|c|c|}
\hline $\begin{array}{c}\text { Variables } \\
\text { dependientes }\end{array}$ & Coeficientes & $\mathrm{T}$ & $\begin{array}{l}\text { Varianza } \\
\text { del error }\end{array}$ & $\mathrm{R}^{2}$ \\
\hline $\begin{array}{l}\text { COGNITIVA } \\
\text { • Publicidad }\end{array}$ & 0.51 & 8.36 & 0.75 & 0.27 \\
\hline $\begin{array}{l}\text { AFECTIVA } \\
\text { - Cognitiva } \\
\text { - Publicidad } \\
\end{array}$ & $\begin{array}{l}0.58 \\
0.29 \\
\end{array}$ & $\begin{array}{l}9.32 \\
4.49 \\
\end{array}$ & 0.51 & 0.49 \\
\hline $\begin{array}{l}\text { CONATIVA } \\
\cdot \text { Afectiva }\end{array}$ & 0.69 & 11.14 & 0.48 & 0.42 \\
\hline
\end{tabular}

La hipótesis 3 trata de analizar la influencia de la promoción en la secuencia jerárquica decisional de los alumnos. Se evaluó el modelo inicial y los indicadores señalaron que éste presentaba un ajuste medio a los datos disponibles ( $\mathrm{SRMR}=0.073$; $\mathrm{GFI}=0.89 ; \mathrm{AGFI}=0.86 ; \mathrm{CFI}=0.91 ; \mathrm{IFI}=0.91$ ). Se revisó la literatura para analizar si existían fundamentos teóricos para explicar el ajuste medio encontrado y agregar nuevas relaciones entre las variables. El nivel medio se podría explicar porque las promociones pueden disminuir la percepción de calidad de los productos o servicios (Raghubir, 2004 a,b) y por ello, en el sector educativo su uso puede ser cuestionado por decisores racio- 
nales. En cuanto a nuevas relaciones, varios autores (ver Eichenbaum y Bodkin, 2000; Gordon y Ford-Hutchinson, 2002) señalan que se debería revisar el impacto del componente afectivo en la secuencia decisional ya que este componente puede condicionar la aceptación de los mensajes promocionales. Igualmente, Young (2004) concluye que la emoción puede condicionar las respuestas cognitivas y por tanto impactar en la influencia de la comunicación sobre la intención de compra o variable conativa. Después de incorporar esta relación, se encontró que el nuevo modelo ofrecía un mejor ajuste $(\mathrm{SRMR}=0.052 ; \mathrm{GFI}=0.93 ; \mathrm{AGFI}=0.91 ; \mathrm{CFI}=0.96 ; \mathrm{IFI}=0.96)$. Así, los índices y parámetros mostrados en la tabla 9 , confirman que a mayor promoción, se encontrará un mayor nivel de los componentes cognitivo, afectivo y conativo, aunque mediado por la influencia de la promoción en la variable afectiva.

Tabla 9. Resultados del modelo final de la influencia de la Promoción

\begin{tabular}{|c|c|c|c|c|}
\hline $\begin{array}{c}\text { Variables } \\
\text { dependientes }\end{array}$ & Coeficientes & $\mathrm{T}$ & $\begin{array}{l}\text { Varianza } \\
\text { del error }\end{array}$ & $\mathrm{R}^{2}$ \\
\hline $\begin{array}{l}\text { COGNITIVA } \\
\text { • Promoción }\end{array}$ & 0.67 & 8.07 & 0.32 & 0.38 \\
\hline $\begin{array}{l}\text { AFECTIVA } \\
\text { - Cognitiva } \\
\text { - Promoción }\end{array}$ & $\begin{array}{l}0.56 \\
0.69\end{array}$ & $\begin{array}{l}5.76 \\
6.55\end{array}$ & 0.41 & 0.36 \\
\hline $\begin{array}{l}\text { CONATIVA } \\
\bullet \text { Afectiva }\end{array}$ & 0.72 & 10.06 & 0.18 & 0.48 \\
\hline
\end{tabular}

La hipótesis 4 predecía que a mayor nivel de relaciones públicas existirá un mayor nivel en las etapas jerárquicas del proceso decisional. Se evaluó el modelo inicial y los indicadores señalaron que nuestro modelo presentaba un bajo ajuste a los datos disponibles (SRMR=0.076; GFI=0.89; AGFI =0.86; CFI=0.90; IFI=0.90). Para incrementar el poder explicativo del modelo se revisó la literatura para analizar si existían fundamentos teóricos para agregar nuevas relaciones entre las variables. Al igual que los casos anteriores, se encontró que la literatura sugiere incorporar al componente afectivo en este modelo. Por ejemplo, las emociones positivas generadas por los mensajes influyen en su efectividad (Dillard y Peck, 2000), en su elección (Smith y Insko, 1987) y respuesta a los mismos (Einwiller et al., 2006). En el caso de decisores con alto involucramiento (como el caso de estudiantes universitarios), la respuesta emocional genera confianza y disminuye la necesidad de procesar mas información (Hallahan, 1999) así como mejora la actitud hacia los anuncios (Goldsmith et al., 2000). 
Después de agregar la relación sugerida por la literatura, el nuevo modelo presentó un mejor ajuste (SRMR=0.059; $\mathrm{GFI}=0.92 ; \mathrm{AGFI}=0.89 ; \mathrm{CFI}=0.95 ; \mathrm{IFI}=0.95)$. En la tabla 10 se exponen los índices y parámetros del modelo final, el cual permite afirmar que a mayor nivel de relaciones públicas, se encontrará un mayor nivel de los componentes cognitivo, afectivo y conativo, pero después de incorporar la influencia de las relaciones publicas en el componente afectivo.

Tabla 10. Resultados del modelo final de la influencia de las Relaciones Públicas

\begin{tabular}{|c|c|c|c|c|}
\hline $\begin{array}{c}\text { Variables } \\
\text { dependientes }\end{array}$ & Coeficientes & $\mathrm{T}$ & $\begin{array}{l}\text { Varianza } \\
\text { del error }\end{array}$ & $\mathrm{R}^{2}$ \\
\hline $\begin{array}{l}\text { COGNITIVA } \\
\text { • Relac. Públicas }\end{array}$ & 0.56 & 7.96 & 0.68 & 0.31 \\
\hline $\begin{array}{l}\text { AFECTIVA } \\
\text { - Cognitiva } \\
\text { - Relac. Públicas }\end{array}$ & $\begin{array}{l}0.44 \\
0.49\end{array}$ & $\begin{array}{l}7.71 \\
7.39\end{array}$ & 0.38 & 0.62 \\
\hline $\begin{array}{l}\text { CONATIVA } \\
\cdot \text { Afectiva }\end{array}$ & 0.60 & 9.86 & 0.64 & 0.36 \\
\hline
\end{tabular}

La hipótesis 5 pretendía validar que hay componentes de comunicación comercial que influyen más que otros en el proceso decisional. De acuerdo al valor de los índices (Cheung y Rensvold, 2002), podemos observar que el CFI del modelo de las relaciones públicas es mayor que el de la publicidad (0.95 vs 0.94$)$. Estas diferencias son apoyadas por la literatura, la cual indica que las relaciones públicas tienen más efecto que la publicidad debido a su credibilidad (Cameron, 1994; Hallahan, 1999; Linning, 2004). Pero, que el modelo de la promoción, después de las modificaciones señaladas, consigue un CFI mayor que las anteriores (0.96). Estos resultados confirman lo señalado por algunos autores, que las empresas tienen un interés creciente por usar esta variable debido a su efectividad en condicionar el proceso de compra (Neslin, 2002) y que los clientes están mas dispuestos a invertir en promociones y relaciones publicas, debido a sus mejores resultados (Hutton, 2002).

En cuanto a la hipótesis 6 , ésta predecía que el uso de los tres componentes o variables de comunicación (comunicación comercial integrada-CCI) tendría un impacto mayor en el proceso jerárquico, que el uso aislado de los mismos. Para facilitar la presentación de resultados, antes de proceder a la validación del modelo -basándonos en la teoría- se creó una variable latente, que integraba las tres variables de comunicación. Esta variable latente (CCI), obtuvo un buen ajuste a los datos (SRMR=0.045; $\mathrm{GFI}=0.95 ; \mathrm{AGFI}=0.93 ; \mathrm{CFI}=0.97 ; \mathrm{IFI}=0.97)$. 
Aunque el modelo inicial de nuestra hipótesis presentó un ajuste aceptable ( $\mathrm{SRMR}=0.065 ; \mathrm{GFI}=0.90 ; \mathrm{AGFI}=0.89 ; \mathrm{CFI}=0.91 ; \mathrm{IFI}=0.91)$, se revisó la literatura para buscar teoría que justificara la inclusión de nuevas relaciones, con el fin de mejorar el ajuste del modelo. Según Gardener y Trivedi (1998), las acciones de comunicación son más efectivas cuando consideran todos los niveles de la respuesta jerárquica. Por tanto, en nuestro caso agregamos una relación directa de la CCI sobre el componente afectivo y conativo. Después de incorporar estas relaciones, los resultados obtenidos $(\mathrm{SRMR}=0.034 \mathrm{GFI}=0.95 \mathrm{AGFI}=0.93 \mathrm{CFI}=0.97 \mathrm{IFI}=0.97)$ confirman la validación de esta hipótesis. La tabla 11 muestra los resultados del modelo final que integra el efecto conjunto de las variables de comunicación comercial, sobre el proceso decisional jerárquico. Señalando que el efecto que tiene sobre el proceso decisional es mayor que el que se obtiene con las variables aisladas $(\Delta \mathrm{CFI}=0.03,0.02,0.01$, respectivamente), pero considerando la influencia directa sobre los componentes afectivo y conativo, tal como lo recomienda la teoría (Gardener y Trivedi, 1998).

Finalmente, nuestros resultados coinciden con investigaciones desarrolladas con otros medios (televisión e impresos) en donde se apoya el efecto teórico de la sinergia de las diversas variables de comunicación (ver Naik y Raman, 2003).

Tabla 11 . Resultados del modelo final de la influencia de la Comunicación Comercial

\begin{tabular}{|l|c|c|c|c|}
\hline $\begin{array}{c}\text { Variables } \\
\text { dependientes }\end{array}$ & Coeficientes & $\mathrm{T}$ & $\begin{array}{c}\text { Varianza } \\
\text { del error }\end{array}$ & $\mathrm{R}^{2}$ \\
\hline $\begin{array}{c}\text { COGNITIVA } \\
\text { - CCI }\end{array}$ & 0.75 & 10.50 & 0.45 & 0.52 \\
\hline $\begin{array}{l}\text { AFECTIVA } \\
\text { - Cognitiva } \\
\text { - CCI }\end{array}$ & 0.61 & 9.85 & 0.41 & 0.59 \\
\hline $\begin{array}{l}\text { CONATIVA } \\
\text { - Afectiva }\end{array}$ & 0.59 & 8.03 & & \\
- CCI & 0.53 & 7.18 & 0.64 & \\
\hline
\end{tabular}

\section{Conclusiones}

El propósito de este artículo ha sido contribuir al desarrollo de la aplicabilidad de la comunicación comercial en un contexto no lucrativo. Para ello hemos profundizado en analizar si si las influencias de estos componentes pueden ser modificadas por factores individuales (e.g. proceso decisional), y/o por factores relacionados con su gestión. 
Por tanto, surgen diferentes tipos de implicaciones para estas disciplinas. En cuanto a la teoría sobre procesos decisionales, nuestra investigación validó que el modelo jerárquico clásico es usado por los decisores de nuestra muestra. Las características específicas de los respondientes (alumnos universitarios) explican que se haya validado este modelo, en comparación con modelos rivales que la teoría sugiere para situaciones de compra con baja diferenciación del producto y bajo involucramiento del consumidor. Así, aunque se trate de un servicio con el que tienen experiencia diaria, sin embargo usan una ruta central de procesamiento de información que se inicia con una interpretación cognitiva de la misma. Estos resultados constituyen un aporte a la literatura de marketing, la cual indica que estos modelos jerárquicos no están suficientemente validados (Huey, 1999; Weilbacher, 2001, 2002) a pesar de su lógica y de que han estado presentes en este tipo de literatura desde hace décadas (Barry, 2002; Weilbacher, 2001).

Nuestros hallazgos apoyan a los autores que desde hace décadas sugirieron que la efectividad de la publicidad es mediada por la dimensión cognitiva y afectiva del consumidor (McGuire, 1978; Lavidge y Steiner, 1961). Sin embargo, los resultados también sugieren que este proceso no es automático y lineal. Es decir, que los decisores no son pasivos en el seguimiento de la secuencia, sino que sus respuestas afectivas ante los estímulos de comunicación pueden condicionar todo el proceso. Esto es reconocido -de manera implícita- por abundante teoría sobre las actitudes hacia la publicidad y hacia la marca. Aquí, desde hace tiempo algunos autores han indicado que las emociones que despierta la publicidad pueden modificar la efectividad de la misma (entre otros, ver Batra y Ray, 1986; Bruner y Kumar, 2000; Mitchell y Olson, 1981). En este sentido, nuestros resultados también podrían contribuir a esa línea de investigación, pues muestra la influencia de la variable afectiva en diferentes variables de comunicación.

Nuestros resultados también validaron que el proceso decisional puede ser estimulado por una variable de comunicación, o por la integración de las mismas. Asimismo, de acuerdo a la teoría, algunas variables tienen un mayor impacto que otras. Pero, también según la teoría, es recomendable usar una mezcla de variables para conseguir mayor impacto en el proceso decisional. En este caso se encontró que la efectividad depende de una estimulación directa del componente afectivo y conativo. Una explicación posible sería que al usar varios estímulos, el decisor trata de filtrar los más importantes, para evitar una sobrecarga de información que puede afectar su memoria temporal y por tanto la comprensión de los mensajes (Just et al., 1996).

El hecho de haber comprobado que las variables de comunicación comercial también son efectivas en el sector educativo, sugiere que nuestra investigación además tie- 
ne implicaciones para los gestores de las universidades. Así, nuestros resultados les proveen información sobre cuales son las variables que deben usar para conseguir mantener el interés institucional de sus alumnos. Asimismo, dado que hemos validado originales medidas de variables publicitarias, éstas les pueden servir como una guía para sus acciones de comunicación. Estas también pueden servirles como una herramienta para diagnosticar su nivel de uso y pronosticar la competitividad institucional, ya que se ha validado su impacto en el proceso decisional de los alumnos. Sin embargo, nuestra investigación también plantea ciertos retos a los gestores universitarios. Estos deben ser creativos para que sus mensajes impacten directamente en el componente afectivo y/o en el conativo, tanto si usan los componentes de manera aislada como si los usan en conjunto. Por creatividad entendemos la habilidad que debe tener el gestor para usar imágenes y sonidos en la formación de mensajes que puedan persuadir a su audiencia. Por tanto, los gestores universitarios deben ser creativos para crear y usar mensajes con cada uno de los componentes de comunicación, de manera que se puedan integrar para generar una sinergia en su capacidad de influencia. Este requisito se hace más importante si se considera la escasa experiencia que tienen aún las universidades con el manejo de técnicas de comunicación comercial (Boffo, 2004).

La principal limitación de nuestra investigación está relacionada con la metodología utilizada para obtener la información. Esta asume las limitaciones propias del uso de escalas de intervalo y del sesgo subjetivo de los informadores cuando responden encuestas auto administradas. Otra limitación está relacionada con la representatividad de la muestra. Aunque nuestra muestra es representativa del centro estudiado, es posible que la investigación en otros centros educativos pueda arrojar otros resultados. Por este motivo, se recomienda cierta cautela con la generalización de estos resultados. Aunque también cabe mencionar que por el tamaño de la misma, así como por la integración de edades y programas diferentes, nuestros resultados se pueden usar como antecedentes para estudios más profundos. Basamos esta afirmación en que se han elaborado escalas que han obtenido índices de fiabilidad aceptables y han permitido la validación de las hipótesis planteadas.

Finalmente, como líneas futuras de investigación se sugiere la incorporación de otras variables al proceso decisional, como por ejemplo el involucramiento. Tal como ha sido sugerido desde hace décadas para el caso de la publicidad (ver Vaughn, 1980). Igualmente, agregar otras formas de medir la efectividad de la comunicación comercial, como por ejemplo el recuerdo y el reconocimiento al evaluar el componente cognitivo. También se recomienda investigar la interacción entre las variables, es decir, si una noticia de la universidad es mayor/o menor cuando esta se apoya con un mensaje publicitario, o una acción promocional. Asimismo, es aconsejable analizar el 
proceso que sigue los decisores para filtrar los mensajes cuando son usados en una campaña integral de comunicación, y saber cuales son los elementos que priorizan y cuales son los que descartan.

Así, esta investigación ha tratado de ayudar a solucionar la problemática competitiva que enfrentan las universidades, presentando guías para mejorar su posicionamiento a través del uso de la comunicación comercial. Asimismo, ha pretendido responder a las demandas teóricas que indicaban que se debería estudiar el efecto de las campañas de comunicación integral y que hasta el presente no se había desarrollado un modelo para estudiar como éstas podían afectar la jerarquía de respuestas del consumidor (Weilbacher, 2001).

\section{REFERENCIAS BIBLIOGRÁFICAS}

Ablett, J. y Slengesol, I.A. (2000) «Education in Crisis: The Impact and Lessons of the East Asian Financial Shock», 1997-99». Published by UNESCO.

Aвraham, M. y Lodish, L. (1987) «Promoter: An Automated Promotion Evaluation System», Marketing Science, 6 (1): 101-123.

- (1990) «Getting the most out of advertising and promotion», Harvard Business Review, 68 (3): 50-63.

Anderson, J. y Gerbin, D. (1988) «Structural Equation Modelling in Practice: A Review and Recommended Two-Step Approach», Psychological Bulletin, 103 (3): 411-423.

Anderson, N. (1981) Foundation of information integration theory. New York: Academic Press.

Arens, W. (1999) Publicidad, 7 edición, Mc Graw-Hill, U.S.A.

Aylesworth, A. y Mackenzie, S. (1998) «Context is key: the effect of programme-induced mood on thoughts about the ad», Journal of Advertising, 27 (2): 17-31.

Balasubramanian, S. (1994) «Beyond Advertising And Publicity: Hybrid Messages And Public Issues», Journal of Advertising, 23 (4): 29-46.

BARRY, T. (1987) «The Development of the Hierarchy of Effects: An Historical Perspective», Current Issues and Research in Advertising, 10: 251-295.

- (2002) «In Defense of the Hierarchy of Effects: A Rejoinder to Weilbacher», Journal of Advertising Research, May/June: 44-47.

BARRY, T. y HowARD, D. (1990) «A Review and Critique of the Hierarchy of Effects», International Journal of Advertising, 9 (2): 121-35.

BATTA, R. y RAY, M. L. (1986) «Affective Responses Mediating Acceptance of Advertising», Journal of Consumer Research, 13 (2): 236-249.

Batra, R.; Myers, J. y AAKer, D. (1996) Advertising Management, Prentice-Hall, $5^{\text {th }}$ edition.

Belch, G. y BelsCh, M. (2004) Advertising and Promotion: An Integrated Marketing Communication Perspective, $5^{\text {th }}$ edition, NY: McGraw Hill/Irwin. 
BenKahla, S. (2006) «A study of the history and use of integrated marketing communications within publications from 1991-2005», Thesis of Master of Science in Journalism.http://kitkat.wvu.edu:8080/files/4577/Benkahla_Shawn_ thesis.pdf

Bennetr, R. y Kottasz, R. (2006) «Promotional message strategies for disability charities' employment services», International Journal of Nonprofit y Voluntary Sector Marketing, 6 (1): 21-32.

Blythe, J. (2000) Marketing Communications, Ed. Prentice Hall.

Boffo, S. (2004) «Universities and Marketing Mass Communication in Italy», Higher Education Policy, 17 (4): 371-381.

Bollen, K. (1989) Structural equations with latent variables. Nueva York: John Wiley and Sons.

Bollen, K. y Long, J. (1992) «Test of structural equation models: Introduction», Sociological Methods and Research, 21(November):123-131.

Bovee, C.; Thill, J.; Dovel, G. y Wood, M. (1995) Advertising Excellence, McGraw Hill, New York.

Bruner II, G. y Kumar, A. (2000) «Web Commercials and Advertising Hierarchy of Effects», Journal of Advertising Research, 40 (1,2): 35-44.

Cameron, G. (1994) «Does Publicity Outperform Advertising: An Experimental Test of the Third-Party Endorsement», Journal of Public Relations Research, 6 (3): 185-207.

Chandon, P.; Wansink, B. y Laurent, G. (2000) «A benefit congruency framework of sales promotion effectiveness», Journal of Marketing, 64 (3): 65-81.

Chang, Y. y Thorson, E. (2004) «Television and Web Advertising Synergies», Journal of Advertising, 33 (2): 75-84.

CHEN, S. (1998) «The Effects of Framing Price Promotion Messages on Consumers Perceptions y Purchase Intentions», Journal of Retailing, 74 (3): 353-372.

Cheney, G. y Christensen, L. (2001) «Public relations as contested terrain: A critical response». In Heath, R. y Vasquez, G (eds.), Handbook of Public Relations Thousand Oaks: Sage, 167-182.

Cheung, G. y Rensvold, R. (2002) «Evaluating goodness-of-fit indexes for testing measurement invariance», Structural Equation Modeling, 9: 233-255.

Christopher, M. y McDonald, M. (1995) Marketing: An introductory text, Mac Millan Press Ltd.

Clark, B. (1998) Creating entrepreneurial universities organizational pathways of transformation. New York: IAU Press.

Clow, K. (2002 ) Integrated Advertising, Promotion \& Marketing Communication, New Jersey: Prentice Hall

Clow, K. y BAAK, D. (2002) Integrated Advertising, Promotion, and Marketing Communications, Prentice Hall.

Cornelissen, J. y Lock, A. (2000) «Theoretical Concept or Management Fad? Examining the Significance of IMC», Journal of Advertising Research, 40 (5): 7-15.

Cutlip, S.; Center, A. y Broom, G. (2000) Effective Public Relations, $8^{\text {th }}$ edition. London: Prentice Hall. 
Dillard, J. y Peck, E. (2000) «Affect and Persuasion: Emotional Responses to Public Service Announcements», Communication Research, 27 (4): 38-68.

Donnelly-Cox, G. y O’Regan, A. (1999) Resourcing Organisational Growth y Development: A typology of Third Sector Service Delivery Organisation. Trinity College Dublin.

Dore, L., y Crouch, G. (2003) «Promoting Destinations: An Exploratory Study of Publicity Programmes Used by National Tourism Organizations», Journal of Vacation Marketing, 9 (2): 137-51.

Drenth, P.; Thierry, H.; Willems, P. y De Wolff, C. (1984) Handbook of Work and Organizational Psychology. Chichester: John Wiley and Sons, Inc.

Duncan, T. (2005) IMC: Using Advertising and Promotion to Build Brands, $2^{\text {nd }}$ edition, New York, NY: McGraw Hill/Irwin.

Duncan, T. y CAYwood, C. (1996) The Concept, Process, and Evolution of Integrated Marketing Communication. In Integrated Communication: Synergy of Persuasive Voices, Thorson, E. and Moore, J. eds., Mahwah, NJ: Lawrence Erlbaum, 13-34.

Dwyer, F. y TANner, J. (2002) Business Marketing, Boston, Mc Graw Hill

Edell, J. y Keller, K. (1999) «Analyzing Media Interactions: The Effects of Coordinated TV-Print Advertising Campaigns», Working Paper No. 99-120, Marketing Science Institute, Cambridge, MA.

Eichenbaum, H. y Bodkin, J, (2000) «Belief and knowledge as Distinct Forms of Memory». In Memory, Brain, and Belief, D. Schacter, and E. Scarry, eds, Cambridge, MA: Harvard University Press, 176-207.

Einwiller, S.; Fedorikhin, A.; Johnson, A. y Kamins, M. (2006) «Enough Is Enough! When Identification No Longer Prevents Negative Corporate Associations», Journal of the Academy of Marketing Science, 34 (2): 185-194.

Fabrigar, L. y Petty, R. (1999) «The Role of the Affective and Cognitive Bases of Attitudes in Susceptibility to Affectively and Cognitively Based Persuasion», Personality and Social Psychology Bulletin, 25 (3): 363-381.

FiLL, C. (2005) Marketing Communications: Engagement, strategies and practice, $4^{\text {th }}$ edition., Prentice Hall Financial Times.

Gardener, E. y Minakshi, T. (1998) «A Communications Framework to Evaluate Sales Promotion Strategies», Journal of Advertising Research, (May/June): 67-71.

Goldsmith, R.; Lafferty, B. y Newell, S. (2000) «The impact of corporate credibility and celebrity credibility on consumer reaction to advertisements and brands», Journal of Advertising, 29 (3): 43-54.

Gordon, W. y Ford-Hutchinson, S. (2002) «Brains and Brands: Re-thinking the Consumer», Admap, 37 (424):47-50.

GRAEFF, T. (1996) «Using promotional messages to manage the effects of bry y self-image on bry evaluations», Journal of Consumer Marketing, 13:4-18.

Gregory, A. (2001) «Public relations and evaluation: does the reality match the rhetoric?», Journal of Marketing Communications, 7: 171-189.

Groth, J. y Dye, R. (1999) «Service quality:perceived value,expectations, shortfalls, and bonuses», Managing Service Quality, 9 (4): 274-285. 
Grunig, J. y Hunt, T. (1984) Managing Public Relations, Fort Worth: Holt, Rinehart and Wilson.

Guadagn, P. y Little, J.C. (1983) «A logit model of brand choice calibrated on scanner data», Marketing Science, 2 (summer): 203-238.

Hallahan, K. (1996) Product publicity: An orphan of marketing research. In Integrated communications: The search of synergy in communication voices, E. Thorson and $\mathrm{J}$. Moore eds., Mahwah, NJ: Lawrence Erlbaum Associates, Inc.,305-330.

- (1999) «It's Not True What They Say About Publicity's «Implied Third-Party Endorsement Effect», Public Relations Review, 25(3):331-350.

Harris, T. (1993), «How MPR Adds Value To Integrated Marketing Communications», Public Relations Quarterly, (Summer), 13-18.

Holloway, D. (1992) «How to select a measurement system that's right for you: tools for assessing the effectiveness of public relations campaigns», Public Relations Quarterly, 37 (3): 15-17.

Huey, B. (1999) «Advertising's Double Helix: A Proposed New Process Model», Journal of Advertising Research, (May/June): 43-51.

Huizingh, E. y Hoekstra, C. (2003) «Why do consumers like websites?», Journal of Targeting Measurement and Analysis for Marketing, 1 (4): 350-361.

Hutron, J. (2002) Marketing communications: integrated theory, strategy and tactics. Pentagram Publishing, New Jersey.

Ilchul, K.; Han, D. y Schultz, D. (2004) «Understanding the Diffusion of Integrated Marketing Communication», Journal of Advertising Research, (March):31-45.

J AEGER, S. (2001) «Incorporating "Health" into Promotional Messages for Apples: A Means-End Theory Approach», Journal of Food Products Marketing: innovations in food advertising, food promotion, food publicity, y food sales promotion, 6 (4): 27 51 .

J IN, H. (2003) «Compounding Consumer Interest: Effects of Advertising Campaign Publicity on the Ability to Recall Subsequent Advertisements», Journal of Advertising, 32 (4): 29-41.

Jo, S. (2004) «Effect of content type on impact: editorial vs. Advertising, Public Relations Review», 30 (4): 503-512.

Jones, J. (1992) How Much is Enough: Getting the Most from your Advertising Dollar, Lexington Books, New York.

JöRESKOG, K. (1979) «A general approach to confirmatory maximum likelihood factor analysis with addendum». In Joreskög, K. and Sörbom, D. Advances in factor analysis and structural equation models. Cambridge, (MA).

- (1993). Testing structural equation models. In Bollen, K. and Long, J. (eds.), Testing structural equation models. Newbury Park, CA: Sage. 294-316.

Just, M. y CARPENTER, P. (1992) «A capacity theory of comprehension: individual differences in working memory», Psychological Review, 99: 122-149.

Keaveney, P. (2001) Marketing to donors. In Keaveney, P. and Kaufman, M. Marketing for the voluntary sector, Kogan Page, London. 
Keller, L. (2001) «Mastering the Marketing Communications Mix: Micro and Macro perspectives on Integrated Marketing Communication Programs». Journal of Marketing Management, 17: 819-847.

Kelloway, E. (1998) Using LISREL for structural equation modeling: A researcher's guide, London: Sage.

Kitchen, P.; Brignell, J.; Li, T. y Jones, G. (2004) «The Emergence of IMC: A Theoretical Perspective», Journal of Advertising Research, (March): 19-30.

Kotler, P. (1996) Dirección de Mercadotecnia, $8^{\text {a }}$ Edición. Prentice-Hall, México.

Krugman, D.; Reid, L.; Dunn, S. y Barban, A. (1994) Advertising. Its role in modern marketing, The Dryden Press.

Lambin, J. (1994) La Recherche Marketing, Ediscience.

Lauzen, M. (1991) «Imperialism and Encroachment in Public Relations», Public Relations Review, 17, (Fall): 245-55.

Lavidge, R. y Steiner, G. (1961) «A Model of Predictive Measurements of Advertising Effectiveness», Journal of Marketing, 25 (6):59-62.

Lilien, G.; Kotler, P. y Moorthy, K. (1992) Marketing Models. Prentice-Hall International Inc. New Jersey.

Lindemann, W. (1993) «An Effectiveness Yardstick to Measure Public Relations Success», Public Relations Quarterly, 31 (1): 7-10.

LinNING, R. (2004) «Abuse and self-abuse: PR and its USP, plausible deniability», Journal of Communication Management, 9 (1): 65-73.

Loda, M. y Coleman, B. (2005) «Sequence matters: A more effective way to use advertising and publicity», Journal of Advertising Research, 45: 362-372.

Low, G. y MонR J. (2000) «Advertising vs sales promotion: a brand management perspective», Journal of Product and Brand Management, 9 (6): 389-414.

McGurre, W. (1978) «An Information Processing Model of Advertising Effectiveness». In Behavioral and Management Science in Marketing, Davis, H. and Silk, A., eds. New York. 216-231.

McKaY, S. (2003) «Adolescent Risk Behaviors and Communication Research», Journal of Language y Social Psychology, 22 (1):74-82.

Michaelson, D. y STACKs, D. (2007) «Exploring the Comparative Communications Effectiveness of Advertising and Public Relations: An Experimental Study of Initial Branding Advantage», Institute for Public Relations, http://www.instituteforpr.org/files/uploads/Michaelson_Stacks.pdf

Mrtchell, A. y Olson, J. (1981) «Are Product Attribute Beliefs the Only Mediator of Advertising Effects on Brand Attitude?», Journal of Marketing Research, 18 (3): 318-332.

Moriarty, S. (1994) «PR and IMC: The Benefits of Integration», Public Relations Quarterly, 39 (3): 38-44.

Munger, J. y Grewal, D. (2001) «The effects of alternative price promotional methods on consumers' product evaluations and purchase intentions», Journal of Product \& Brand Management, 10 (3): 185-197.

Myers, D., (2002) Social Psychology. $7^{\text {th }}$ edition. McGraw-Hill, Inc., New York. 
NAIK, P. y RAman, K. (2003) «Understanding the Impact of Synergy in Multimedia Comparisons», Journal of Marketing Research, 60 (November): 375-388.

O’Guinn, T.; Allen, C. y Smenik, R. (1990) Publicidad. International Thomson Editors.

ONG, B.; Ho, F. y TRIPP, T. (1997) «Consumer perceptions of bonus packs: an exploratory analysis», Journal of Consumer Marketing, 14 (2): 102-112.

Pelsmacker P.; Geuens, M. y Anckaert, P. (2002) «Media context and advertising effectiveness: the role of context style, context quality and context-ad similarity», Journal of Advertising, 31 (2): 49 -61.

Peterson, M. y Dill, D. (1997) «Understanding the competitive environment of the postsecondary knowledge industry». In Peterson, M., Dill, D., and Mets, L. (eds.) Planning and Management for a Changing Environment. San Francisco: Jossey-Bass.

Phillips, D. (2001) Online Public Relations. London: Kogan Page.

Pickton, B. (2001) Integrated marketing communications, Essex: Pearson Education Ltd.

PrIYA, R. (2004) «Free Gifts with Purchase: Promoting or Discounting Brands?», The Journal of Consumer Psychology, 14 (1 y 2): 181-186.

Pullig, C.; Netemeyer, R. y Biswas, A. (2006) «Attitude Basis, Certainty, y Challenge Alignment: A Case of Negative Brand Publicity», Journal of the Academy of Marketing Science, 34 (4): 528-542 .

Raghubir, P. (2004a), «Coupons in Context: Discounting Prices or Decreasing Sales?», Journal of Retailing, 80 (1): 1-12.

- (2004b), «Free Gift with Purchase: Promoting or Discounting the Brand?», Journal of Consumer Psychology, 14 (1 \& 2): 181-185.

Ralph, S. y Boxall, K. (2005) «Visible Images of Disabled Students: An Analysis of UK University Publicity Materials», Teaching in Higher Education, 10 (3): 371-385 .

RAY, H. (1994) «Advertising and Public Relations in Transition from Communism: The Case of Hungary 1989-1994», Public Relations Review, 20 (4): 357-72.

Ray, M.; Sawyer, A.; Rothschild, M.; Heeler, R.; Strong, E. y Reed, J. (1973) Marketing Communications and Hierarchy of Effects, New Models for Mass Communication Research, Beverly Hills: Sage Publications: 147-176.

Renkema, J. y Hoeken, H. (1998) «The Influence of Negative Newspaper Publicity on Corporate Image in the Netherlands», Journal of Business Communication, 35 (4): 521-535.

RIES, A. y RIES, L (2002) The Fall of Advertising and the Rise of PR, New York, Harper Business.

Rivera, J. y De Juan, M.D. (2001) La promoción de ventas, variable clave del marketing. Ed. ESIC.

Rogers, E. (1962) Diffusion of Innovations (1st edition). London. The Free Press.

Rossiter, J. y PerCy, L. (1997) Advertising \& Promotion Management, $2^{\text {nd }}$ edition, New York NY. McGraw-Hill.

Russell, H. (1961) Defining Advertising Goals for Measured Advertising Results. New York, NY: Association of National Advertisers.

SCHOLten, M. (1996) «Lost and found: the information-processing model of advertising effectiveness», Journal of Business Research, 37: 97-104. 

JERÁRQUICO: UNA EVALUACIÓN EMPÍRICA EN EL CONTEXTO EDUCATIVO

Schultz, D. y Kitchen, P. (2000) «A Response to 'Theoretical Concept or Management Fashion?,» Journal of Advertising Research, 40 (5): 17-21.

Schultz, D.; Tannenbaum, S. y Lauterborn, R. (1993) Integrated Marketing Communications: Pulling It Together and Making It Work, Lincolnwood, IL: NTC Business Books.

Sen, S. y Bhattacharya, C. (2001) «Does Doing Good Always Lead to Doing Better? Consumer Reactions to Corporate Social Responsibility», Journal of Marketing Research, 38 (May): 225-43.

Sethuraman, R. y Teluis, G. (2002) «Does manufacturer advertising suppress or stimulate retail price promotions? Analytical model and empirical analysis», Journal of Retailing, 78 (4): 253-263.

Sharma, A. (2000) «Recall of Television Commercials as a Function of Viewing Context: The Impact of Program-Commercial Congruity on Commercial Messages - Statistical Data Included», Journal of General Psychology, 127 (4): 383-396.

Shir-Lun, A. y Nelson, R. (2006) «The Effects of Identical Versus Varied Advertising and Publicity Messages on Consumer Response», Journal of Marketing Comunications, 12 (2): 109-123.

Shimp, T. y Deloizier, W. (1986) Promotion Management and Marketing Comunications Hinsdale, III.: Dryden.

Simon, H. (1992) «Introduction». In Economics, Bounded Rationality and the Cognitive Revolution. Egidi, M. and Marris, R., eds., Edward Elgar.

Sinkovics, R. y Schlegelmilch, B. (2000) «Marketing Academics in Austria, Germany and Switzerland: Humboldt's Ideals Give Way to Performance Pressure», Journal of Marketing Management, (16): 745-759.

$\mathrm{S}_{\text {мIтH, }}$ P. (1993) Marketing Communications and integrated approach, $2^{\text {th }}$ edition, Kogan Page.

Smith, R. e Insko, C. (1987) «Social Comparison Choice During Ability Evaluation The Effects of Comparison Publicity, Performance Feedback, and Self-Esteem», Personality and Social Psychology Bulletin, 13 (1): 111-122.

SMith, R. y SwINYARD, W. (1982) «Information response models: An integrated approach», Journal of Marketing, (46): 81-93.

Sмiтh, R. y Vogt, C. (1995) «The effects of integrating advertising and negative word-ofmouth communications on message processing and response», Journal of Consumer Psychology, 4 (2): 133-151.

SwaIn, W. (2004) «Perceptions of IMC After a Decade of Development: Who's at the Wheel, and How Can We Measure Success?», Journal of Advertising Research, (March): 46-65.

Telus, G. (1998) Advertising and Sales Promotion Strategy, Reading, MA: Addison-Wesley.

Thorsten, H. (2007) «Assessing EU strategies for publicity», Journal of European Public Policy, 14 (4): 563-581.

TVersky, A. y KahNeman, D. (1974) «Judgement under uncertainty: heuristics and biases», Science, (185): 1124-1131. 
Vakratsas, D. y Ambler, T. (1999) «How Advertising Works: What We Really Know?», Journal of Marketing, 63 (January): 26-43.

Van Reijmersdal, E.; NeiJens, P. y Smit, E. (2005) «Readers' reactions to mixtures of advertising and editorial content in magazines», Journal of Current Issues and Research in Advertising, 27: 39-53.

VAughN, R. (1980) «How Advertising Works: A Planning Model», Journal of Advertising Research, 20 (5): 27-33.

Watson, S. y Barton, A. (1990) Advertising. Its role in modern marketing, The Dryden Press.

Weilbacher, W. (2001) «Does Advertising Cause a 'Hierarchy of Effects'?», Journal of Advertising Research, 41 (6): 19-26.

- (2002) «Weilbacher Comments on In Defense of the Hierarchy of Effects», Journal of Advertising Research, (May/June): 48-49.

Weiner, M. y Bartholomew, D. (2006) Dispelling the myth of PR multipliers and other inflationary audience measures. Gainesville, FL: Institute for Public Relations (www.instituteforpr.org).

Wells, W.; Burnett, J. y Moriarty, S. (2003) Advertising Principles and Practice, $6^{\text {th }}$ edition., Upper Saddle River, NJ: Prentice Hall.

YI, Y. (1990) «Cognitive and affective priming effects of the context for print advertisements», Journal of Advertising, 19 (2): 40-48.

Young, C. (2004) «Capturing the Flow of Emotion in Television Commercials: A New Approach», Journal of Advertising Research, (June): 202-209. 